\title{
常重力和低重力条件下气液两相流实验研究 ${ }^{*}$
}

\author{
赵建福 解京昌 林 海 胡文瑞 \\ (中国科学院力学研究所国家微重力实验室. 北京 100080)
}

\begin{abstract}
摘要实验研究了常重力和低重力条件下水平方管内水气两相流型特征及其相互转 换条件，实验中均观察到了泡状流、弹状流、弹一环状过度流及环状流等流型. 发展了半 理论 Weber 数模型以计入截面形状对弹状流-环状流转换的影响, 较好预测了实验观测 结果. 此外, 滑移流率模型可成功预测大 Froude 数水平两相泡状流-弹状流间的转换边界.
\end{abstract}

\section{关链词微血力 气液两相流 流型 非圆形管道}

气液两相流现象不仅广泛应用在地面常重力环境中的许多工业过程(如石油、化工、能源 及动力等), 而且在空间微重力环境也有很多重要应用, 如主动热控系统、动力环路及深冷液 体的贴存与输送等. 此类系统设计与运行的可靠性, 依赖于对气液两相流机理的充分认识. 流 型因其对两相流压降及传热传质特性等的重要影响, 一直是气液两相流研究中颇受重视的基 础课题之一.

目前, 关于气液两相流型产生与转换机理的知识往往基于地面常重力环境中大量精心设 计的实验的结果. 由于地面常重力环境中, 重力作为主导因素所引起的浮力分层及相间滑移 等复杂影响难以完全揭示, 现有知识远非完善. 空间微重力环境中, 重力作用被消弱甚至完全 消失, 气液两相流具有与常重力环境相比大为简单的特征. 因此, 微重力气液两相流研究将有 助于彻底揭示流型产生与转换的机理, 保证相关技术的顺利发展.

近十多年来, 微重力气液两相流研究的优越性及其在空间应用方面的直接需要, 激励了 微重力气液两相流研究的迅速发展 ${ }^{[1]}$. 不过, 现有微重力气液两相流实验往往采用圆形截面流 道. 非圆形截面流道因更强的截面环流作用使得强化传热成为可能, 更应得到重视. Wölk 等 人 $^{[2]}$ 研究了地面常重力和短时微重力(落塔)条件下的水气两相流现象, 观察了常重力垂直向上 泡状流、弹状流和混块状流及微重力泡状流和弹状流.

本文研究水平方管内水气两相流在常重力和低重力条件下的流型特征, 主要目标是重力 和截面形状对流型产生与转换的影响, 揭示流型转换的机制.

\section{1 实验装置}

实验装置(图 1)是针对俄罗斯 IL-76 失重飞机搭载实验要求研制的. 该装置同时还可以在 地面进行常重力条件下的气液两相流实验研究.

实验介质为水和空气. “分离器-百-调节阀-流量计-混合器-实验段-分离器”形成封闭水相 环路, 流量计可同时测量水流量(量程 $0 \sim 1500 \mathrm{~kg} / \mathrm{h}$, 精度 $0.15 \%$ 全量程)和温度(量程 $0 \sim 200^{\circ} \mathrm{C}$ ). 两个气流测控支路均有一个电磁闹和气体质量流量控制器, 量程分别为 $0 \sim 100$ SLM(标准状

*国家自然科学基金(批准号: 19789201)和“登”计划(编号: 95-预-34)资助项目 
态下升每分钟)和 $0 \sim 10$ SLM, 精度均为 $1.5 \%$ 全量程. 若气流量大于 $10 \mathrm{SLM}$, 大流量支路开启, 小流量支路关闭. 反之, 则开启小流量支路, 关闭大流量支路. 空气在分离单元内从水中分离 出来, 直接排人机舱.

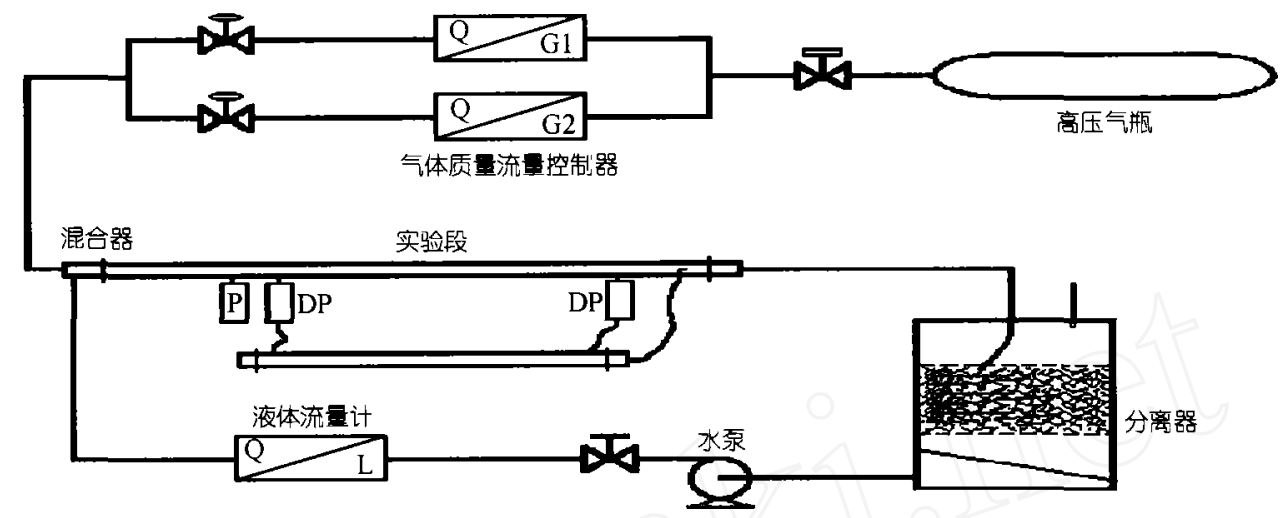

图 1 实验装置简图

混合器内形成的均匀水气混合物进人实验段, 其出口和实验段截面形状相同, 均为 12 $\mathrm{mm} \times 12 \mathrm{~mm}$. 实验段长 $960 \mathrm{~mm}$, 内壁为透明有机玻璃. 观察窗起点距混合器出口 $640 \mathrm{~mm}$, 长 $150 \mathrm{~mm}$. 水气两相流型用 $\mathrm{Hi} 8$ 摄像机记录, 快门速度设定为 $1 / 4000 \mathrm{~s}$ 以减小图像的模糊程度. 距混合器出口 $180 \mathrm{~mm}$ 处安装有绝压传感器(量程 $0 \sim 300 \mathrm{kPa}$ ), 两个差压传感器(量程 $0 \sim 20$ $\mathrm{kPa}$ )分别安装在距混合器出口 300 和 $810 \mathrm{~mm}$ 处, 传感器精度均为 $0.25 \%$ 全量程. 两个差压传 感器的参考端均连接在一个充满水的压力旁管上, 后者通过安装在实验段出口前 $80 \mathrm{~mm}$ 处的 后向皮托管与实验段相连, 以避免在飞行实验中差压传感器发生严重过载. 实验段内沿程压 降由两差压传感器输出信号相减得出.

\section{2 结果与讨论}

\section{1 流型特征}

具体实验条件列于表 1. 根据常用分类办法, 在不同重力实验中均观察到了泡状流、弹状 流、弹-环状过度流和环状流等 4 种流型(图 2).

常重力和低重力流型的显著差异在于: 低重力流型相对于流道中心具有一定的对称性, 而常重力条件下气相在浮力作用下偏于流道上部. 此外, 低重力条件下弹状流中气团前端呈半球 (或椭球)状, 界面相当光滑. 而常重力条件下界面十分粗粘, 存在较大幅度的不规则界面波.

表 1 常重力与低重力实验条件

\begin{tabular}{ccccc}
\hline 气相表观流速 $/ \mathrm{m} \cdot \mathrm{s}^{-1}$ & 液相表观流速 $/ \mathrm{m} \cdot \mathrm{s}^{-1}$ & 压力 $/ \mathrm{kPa}^{-1}$ & 温度 $/{ }^{-} \mathrm{C}$ & 重力水平 \\
\hline $0.08 \sim 6.73$ & $0.32 \sim 1.89$ & $107 \sim 287$ & $29.4 \sim 50.8$ & $1 \mathrm{~g}$ \\
$0.12 \sim 7.99$ & $0.16 \sim 1.06$ & $81 \sim 284$ & $34.2 \sim 44.9$ & $<0.04 \mathrm{~g}$ \\
\hline
\end{tabular}

弹-环状流具有类似于环状流的特征，但流道内偶尔会出现一些难以分辨的泡沫状结构, 将连续的核心气流隔断, 形成类似于弹状流中大气团交替出现的间歇状流动结构. 弹-环状流 是否属于气液两相流基本流型, 目前还存在颇多争议, 随后的讨论假定它是弹状流和环状流 间的过渡流型。 

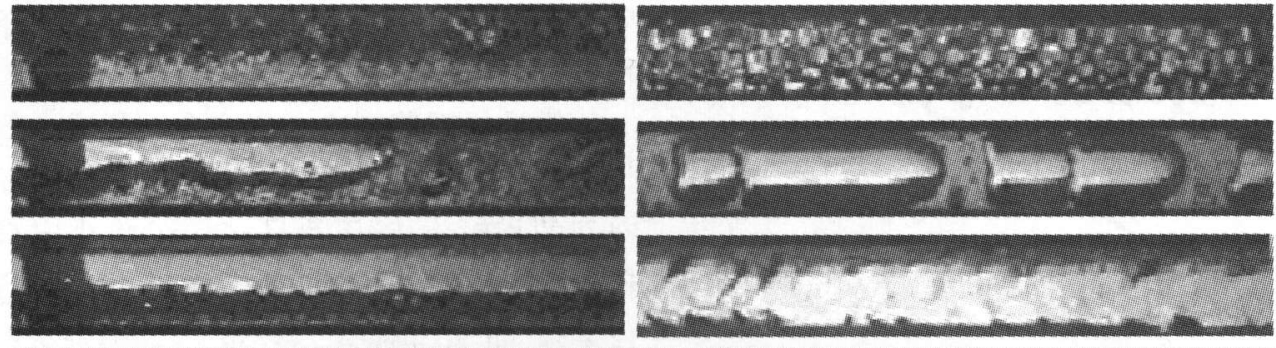

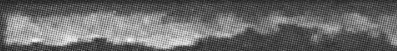

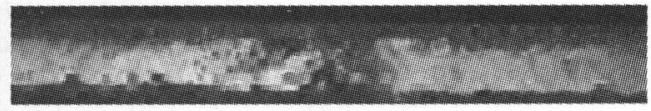

图 2 常重力(左)和低重力(右)气液两相流典型流型特征

自上至下分别为泡状流、弹状流、环状流和弹-环状流，流动方向自左至右

\section{2 泡状流-弹状流转换}

对于泡状流和弹状流, 气液两相速度关系常用滑移流率模型描述 ${ }^{[3]}$ :

$$
U_{\mathrm{G}}=C_{0} U_{\mathrm{M}}+U_{0},
$$

其中, $U_{\mathrm{G}}=U_{\mathrm{SG}} / \varepsilon$ 和 $U_{\mathrm{M}}=U_{\mathrm{SG}}+U_{\mathrm{SL}}$ 分别代表气相平均速度和混合速度, $\varepsilon, U_{\mathrm{SG}}$ 和 $U_{\mathrm{SL}}$ 分别代 表气相空隙率和气液两相表观速度. 气相分布常数 $C_{0}$ 和气泡自由上升速度 $U_{0}$ 为 2 个经验参 数 ${ }^{[4 \sim 6]}$.

在微重力或 Froude 数 $F r=U_{\mathrm{M}} / \sqrt{g D}>3.5$ (式中 $D$ 为流道内径) 时, 可以忽略 $U_{0}$ 的影响 ${ }^{[7]}$, (1) 式可改写为

$$
U_{\mathrm{SL}}=\frac{1-C_{0} \varepsilon}{C_{0} \varepsilon} U_{\mathrm{SG}} .
$$

按滑移流率模型或空隙率模型的观点 ${ }^{[8]}$, 控制微重力两相流动由泡状流向弹状流转换的机制 是气泡间的合并, 而后者依赖于气泡的装填程度即气相空隙率的大小, 因此存在一临界空隙 率 $\varepsilon_{\mathrm{c}}$, 超过该值两相流即由泡状流转换为弹状流. 这样, (2)式中空隙率改为 $\varepsilon_{\mathfrak{c}}$, 即为相应条件 下泡状流-弹状流的转换条件.

Colin 等人 ${ }^{[9]}$ 定义转换参量 $X_{\mathrm{C}}=C_{0} \varepsilon_{\mathrm{c}}$, 并根据微重力实验结果归纳出:

$$
\begin{aligned}
& \text { 当 } S u<1.5 \times 10^{6} \text { 时, } X_{\mathrm{C}} \approx 0.54 \text {, } \\
& \text { 当 } S u<1.7 \times 10^{6} \text { 时, } X_{\mathrm{C}} \approx 0.24 \text {, }
\end{aligned}
$$

其中, Suratman 数 $S u=\sigma D / \rho_{\mathrm{L}} v_{\mathrm{L}}{ }^{2}, \sigma, \rho_{\mathrm{L}}$ 和 $v_{\mathrm{L}}$ 分别代表表面张力、液相介质的密度和运动黏性系 数. Jayawardena 等人 ${ }^{[10]}$ 利用和 Colin 等人几乎完全相同的实验结果, 得到一个新的泡状流-弹 状流转换模型, 整理可得

$$
X_{\mathrm{C}}=K_{1} \frac{v_{\mathrm{G}}}{v_{\mathrm{L}}} /\left(K_{1} \frac{v_{\mathrm{G}}}{v_{\mathrm{L}}}+S u^{2 / 3}\right),
$$

其中, $v_{\mathrm{G}}$ 代表气相介质的运动黏性系数, 经验参数 $K_{1}=464.16$. 本实验中 Suratman 数 $S u \approx(1.95$ $\pm 0.35) \times 10^{6}$, 据 $(5)$ 式可得 $X_{\mathrm{C}}=0.45 \pm 0.03$, 和实验拟合结果 $X_{\mathrm{C}}=0.44$ 符合甚好.

此外, 根据 Bendiksen ${ }^{[7]}$ 实验结果, $F r>3.5$ 时, (2)式对常重力水平气液两相泡状流和弹状 流仍然成立, 滑移流率模型将仍然适用. 不过, 由于气相在浮力作用下往往集中于流道上部, 导致在较小的截面平均的空隙率时, 局部空隙率即超过临界值, 产生大气团. 因此, 常重力条 件下转换参量必然小于低重力时的数值. 图 3 虚线为常重力条件下 $F r>3.5$ 时的实验拟合结 


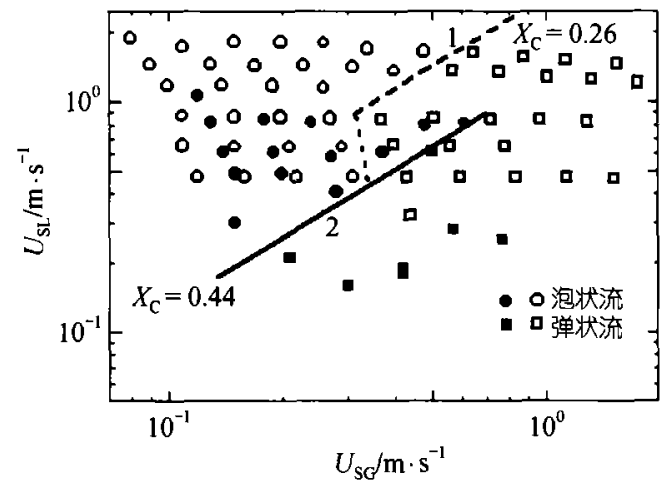

图 3 气液两相泡状流-弹状流间的转换 空心符号示常重力, 实心符号示低重力, 1 示实验观测 边界, 2 示滑移流率预测边界
果, 转换参数为 $X_{\mathrm{C}}=0.26$, 约为低重力条件下 相应数值的一半.

假设局部范围内小气泡合并形成大气团的 临界空隙率近似为常数, 流道内气相实际存在 空间的相对大小(用符号 $\varphi$ 表示)将决定截面平 均的临界空隙率的数值, 转换参数 $X_{\mathrm{C}}$ 将近似正 比于 $\varphi$, 即 $X_{\mathrm{C}}=\varphi X_{\mathrm{C} 0}$ (这里, $X_{\mathrm{C} 0}$ 表示微重力条 件下的转换参数值). 本实验中, 常重力泡状流 一弹状流转换边界附近, 出现气泡的下缘约在流 道中心, $\varphi \approx 1 / 2$. 转换参数的拟合值表明上述 推论是正确的. 如何将滑移流率模型扩展以包 括 $F r<3.5$ 的情形, 将是今后需解决的问题.

\section{3 弹状流-环状流转换}

目前关于气液两相弹状流-环状流转换机制的假说相当多 ${ }^{[1]}$. 比较表明, Zhao 等人 ${ }^{[11]}$ 提出 的半理论 Weber 数模型预测的弹状流-环状流转换边界和现有微重力条件下, 圆截面直管道内 不同实验介质和管径条件下气液两相流实验数据符合程度最好, 甚至对地面常重力环境中利 用中性悬浮液液两相流与毛细管气液两相流模拟微重力气液两相流实验结果也符合甚好, 表 明该模型正确体现了弹状流-环状流转换的机制. 为了计人不同流道截面形状的影响, 这里需 对该模型加以扩展.

半理论 Weber 数模型 ${ }^{[11]}$ 的基本假设是: 控制弹状流-环状流转换的根本机制在于气流惯性 冲击力和表面张力间平衡的破坏. 考虑到截面形状的变化, 方形截面流道内气相惯性冲击力 可表示为 $F_{\mathrm{I}}=D^{2} \rho_{\mathrm{G}} U_{\mathrm{SG}}\left(U_{\mathrm{G}}-U_{\mathrm{L}}\right)$ (这里, $D$ 表示流道截面边长), 作用于气液界面上的表面张力 可表示为 $F_{\mathrm{S}}=2 \pi R_{\mathrm{B}} \sigma$ (其中, $R_{\mathrm{B}}$ 表示弹状流-环状流转换边界附近气核半径或气团前端半径). 几何关系考虑可得 $\pi R_{\mathrm{B}}^{2} / D^{2}=\varepsilon$. 假设滑移流率关系对弹状流-环状流转换边界附近的弹状流仍 然成立, 则弹状流-环状流转换条件可表示为

$$
\begin{gathered}
U_{\mathrm{SG}}=\sqrt{\frac{2 \sqrt{\pi} \kappa C_{0} \sigma \varepsilon^{3 / 2}(1-\varepsilon)}{\left(C_{0}-1\right) \rho_{\mathrm{G}} D},} \\
U_{\mathrm{SL}}=\frac{1-C_{0} \varepsilon}{C_{0} \varepsilon} U_{\mathrm{SG}},
\end{gathered}
$$

其中, $\boldsymbol{\kappa}$ 为一量级为 1 的经验参数, 代表发生弹状流-环状流转换时气相惯性冲击力与表面张力 的相对比值. 该模型还可以用气、液两相表观 Weber 数 $\mathrm{We}_{\mathrm{SI}}=\rho_{1} U_{\mathrm{SI}}{ }^{2} D / \sigma$ (其中, 下标 $\mathrm{I}=\mathrm{G}$ 或 $\mathrm{L}$ 分别代表气相或液相)表示为无量纲的形式(这正是其名称的来历). 和圆形截面流道 ${ }^{[11]}$ 相比, (6)式右端项系数由 4 变为 $2 \sqrt{\pi}$, 反映了截面形状的影响.

图 4 所示半理论 Weber 数模型预测取经验参数 $\kappa$ 为 1 , 气相分布常数 $C_{0}$ 取为 1.35 (方形截 面流道 $)^{[2]}$. 可以看到, 考虑了截面形状效应的半理论 Weber 数模型的预测结果, 不仅和低重力 实验数据符合较好, 而且和常重力实验结果的符合也令人满意, 表明半理论 Weber 数模型同样 正确反映了常重力弹状流-环状流转换的机制. 对实验条件深人分析表明, 常重力弹状流-环状 流转换边界附近, Froude 数均大于 6, 满足 $F r>3.5$ 的条件, 因此, (2)式对常重力水平气液两相 
弹状流仍然成立. 由于不同重力条件下气相分 布参数变化不大, 该模型与不同重力实验结果 都符合甚好当属必然.

\section{3 结论}

本文实验研究了常重力和低重力条件下方 形截面管道内水气两相水平流动的流型特征及 其相互转换条件. 在所研究的气液两相流量范 围内, 不同重力条件下的实验中均观察到了泡 状流、弹状流、弹-环状过度流和环状流等 4 种 流型.

本文发展了半理论 Weber 数模型, 以便计 人非圆形流道截面的形状效应. 实验数据和模

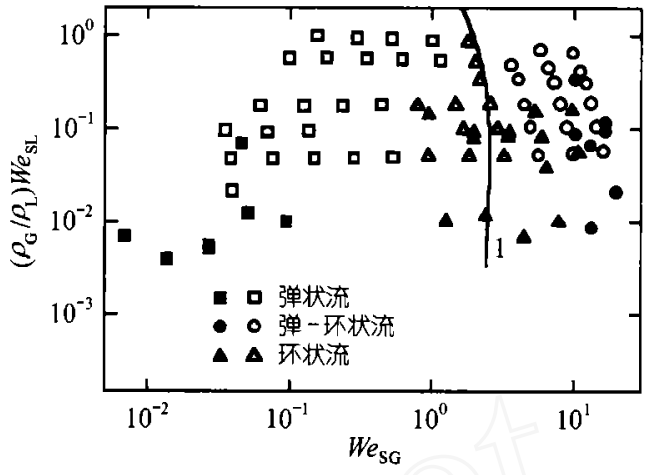

图 4 气液两相弹状流-环状流间的转换 空心符号示常重力, 实心符号示低重力, 1 示半理论 Weber 数模型 型预测结果的比较表明, 半理论 Weber 数模型正确反映了大 Froude 数水平气液两相弹状流环状流转换的机制, 较好地预测了不同重力条件下实验观察到的弹状流-环状流转换边界.

对泡状流-弹状流转换的分析表明，控制该转换的机制是气泡间的合并. 在大 Froude 数水 平气液两相流动中, 描述泡状流-弹状流转换边界的数学方程具有相同的形式, 不同重力条件 下的转换参数的具体数值正比于流道内气相实际存在空间的相对大小及微重力条件下的转换 参数. 因此, 微重力实验可作为确定不同重力条件下大 Froude 数水平气液两相泡状流-弹状流 转换参数的基础. 对于小 Froude 数水平气液两相流动, 尚需进行更深人的研究.

致谢中国科学院空间科学与应用研究中心张玉涵研究员和吕从民工程师在实验过程中 提供了极大的帮助，谨致谢意.

\section{参考文献}

1 赵建福. 微重力条件下气液两相流流型研究进展. 力学进展, 1999, 29: 369 382

2 Wölk G, Dreyer M, Rath H J. Investigation on two-phase flow in small diameter non-circular channels under low and normal gravity. In: Space Technology \& Applications International Forum-1999. Albuquerque: AIP-Conference 458, 1999. S785 S791

3 Zuber N, Findlay J A. Average volumetric concentration in two-phase flow systems. J Heat Transfer, 1965, 87: 453 468

4 Fabre J, Liné A. Modeling of two-phase slug flow. Ann Rev Fluid Mech, 1992, 24: $21 \sim 46$

5 Hewitt G F. Multiphase flow: the gravity of the situation. In: $3^{\text {rd }}$ Microgravity Fluid Physics Conf. Cleveland, NASA CP-3338, 1996. $1 \sim 23$

6 Mishima K, Ishii M. Flow regime transition criteria for upward two-phase flow in vertical tubes. Int J Heat Mass Transfer, 1984, 27(5): $723 \sim 737$

7 Bendiksen $\mathrm{K} \mathrm{H}$. An experimental investigation of the motion of long bubbles in inclined tubes. Int J Multiphase Flow, 1984, 10: $467 \sim 483$

8 Dukler A E, Fabre J A, McQuillen J B, et al. Gas-liquid flow at microgravity conditions: flow patterns and their transitions Int J Multiphase Flow, 1988, 14(4): $389 \sim 400$

9 Colin C, Fabre J, McQuillen J. Bubble and slug flow at microgravity conditions: state of knowledge and open questions. Chem Eng Comm, 1996, 141/142: $155 \sim 173$

10 Jayawardena S S, Balakotaiah V, Witte L C. Flow pattern transition maps for microgravity two-phase flow. AIChE J, 1997, 43: $1637 \sim 1640$

11 Zhao J F, Hu W R. Slug to annular flow transition of microgravity two-phase flow. Int J Multiphase Flow, 2000, 26: 1295 1304 